\title{
Invariants for Discrete Structures - An Extension of Haar Integrals over Transformation Groups to Dirac Delta Functions
}

\author{
Hans Burkhardt ${ }^{1}$, Marco Reisert ${ }^{1}$, and Hongdong $\mathrm{Li}^{2}$ \\ 1 University of Freiburg, Computer Science Department, \\ 79110 Freiburg i.Br., Germany \\ \{burkhardt, reisert\}@informatik. uni-freiburg.de \\ 2 National ICT Australia (NICTA), Dpt. of Sys. Eng., Australian National \\ University (ANU), Canberra ACT, Australia \\ hongdong.li@anu.edu.au
}

\begin{abstract}
Due to the increasing interest in $3 \mathrm{D}$ models in various applications there is a growing need to support e.g. the automatic search or the classification in such databases. As the description of $3 \mathrm{D}$ objects is not canonical it is attractive to use invariants for their representation. We recently published a methodology to calculate invariants for continuous $3 \mathrm{D}$ objects defined in the real domain $\mathbb{R}^{3}$ by integrating over the group of Euclidean motion with monomials of a local neighborhood of voxels as kernel functions and we applied it successfully for the classification of scanned pollen in $3 \mathrm{D}$. In this paper we are going to extend this idea to derive invariants from discrete structures, like polygons or 3D-meshes by summing over monomials of discrete features of local support. This novel result for a space-invariant description of discrete structures can be derived by extending Haar integrals over the Euclidean transformation group to Dirac delta functions.
\end{abstract}

\section{Introduction}

Invariant features are an elegant way to solve the problem of e.g. space invariant recognition. The idea is to find a mapping $T$ which is able to extract intrinsic features of an object, i.e., features that stay unchanged if the object's position and/or orientation changes. Such a transformation $T$ necessarily maps all images of an equivalence class of objects under the transformations group $G$ into one point of the feature space:

$$
\mathbf{x}_{1} \stackrel{G}{\sim} \mathbf{x}_{2} \quad \Rightarrow \quad T\left(\mathbf{x}_{1}\right)=T\left(\mathbf{x}_{2}\right) \quad
$$

A mapping $T$ which is invariant with respect to $G$ is said to be complete if $T$ is a bijective mapping between the invariants and the equivalence classes, i.e. if we additionally ask

$$
T\left(\mathbf{x}_{1}\right)=T\left(\mathbf{x}_{2}\right) \quad \Rightarrow \quad \mathbf{x}_{1} \stackrel{G}{\sim} \mathbf{x}_{2} \quad
$$


For a given gray scale image $\mathbf{X}$ and a kernel function $f(\mathbf{X})$ it is possible to construct an invariant feature $T[f](\mathbf{X})$ by integrating $f(g \mathbf{X})$ over the transformation group $G$ :

$$
T[f](\mathbf{X}):=\int_{G} f(g \mathbf{X}) d g .
$$

As kernel functions $f$ we typically use monomials from a local pixel- or voxel-

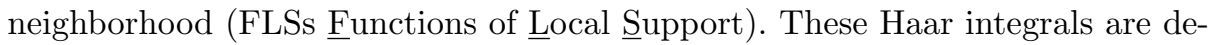
fined for continuous objects in $\mathbb{R}^{2}$ or $\mathbb{R}^{3}([\underline{5,6} 2,3])$. This integral can in practice only be evaluated for compact groups, which means that the parameters describing the group lie in a finite region. In the sequel we will call these invariants HIs (

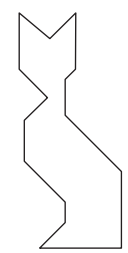

a)

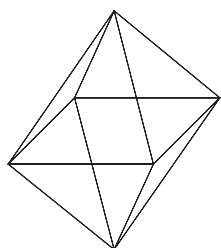

b)

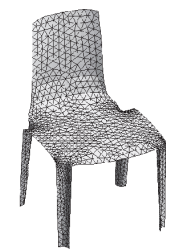

c)

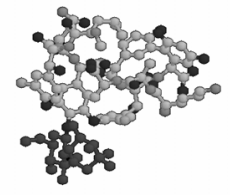

d)

Fig. 1. Discrete structures in 2D and 3D: (a) closed contour described by a polygon (b) wireframe object (c) 3D triangulated surface mesh (d) molecule.

For discrete objects $\boldsymbol{\Delta}$ (see Fig. 1) which vanish almost everywhere this integral delivers, however, trivial or zero results (depending on the kernel function). Discrete structures can be described by Dirac delta functions (examples of distributions or generalized functions), which are different from zero on a set of measure zero of the domain. However, properly chosen integrals of these delta functions exist and deliver finite values. We will use this concept to define proper Haar invariants from discrete structures (DHIs Discrete Haar Invariants).

There exist a vast literature on invariant-based shape representations and object recognition problems; space limitations do not allow here a thorough review. However, to the best of the authors' knowledge there exist no similar approaches to the DHIs.

\section{Invariants for Discrete Objects}

For a discrete object $\boldsymbol{\Delta}$ and a kernel function $f(\boldsymbol{\Delta})$ it is possible to construct an invariant feature $T[f](\boldsymbol{\Delta})$ by integrating $f(g \boldsymbol{\Delta})$ over the transformation group $g \in G$. Let us assume that our discrete object is different from zero only at its vertices. A rotation and translation invariant local discrete kernel function $h$ takes care for the algebraic relations to the neighboring vertices and we can write 


$$
f(\boldsymbol{\Delta})=\sum_{i \in \mathbb{V}} h\left(\boldsymbol{\Delta}, \mathbf{x}_{i}\right) \delta\left(\mathbf{x}-\mathbf{x}_{i}\right),
$$

where $\mathbb{V}$ is the set of vertices and $\mathbf{x}_{i}$ the vector representing vertex $i$.

In order to get finite values from the distributions it is necessary to introduce under the Haar integral another integration over the spatial domain $\mathbf{X}$. By choosing an arbitrary integration path in the continuous group $G$ we can visit each vertex in an arbitrary order the integral is transformed into a sum over all local discrete functions allowing all possible permutations of the contributions of the vertices. As the discrete neighborhood functions are attached to the vertices they are already invariant to $G$, i.e. $h\left(g \boldsymbol{\Delta}, g \mathbf{x}_{i}\right)=h\left(\boldsymbol{\Delta}, \mathbf{x}_{i}\right)$ and hence we get

$$
\begin{aligned}
T[f](\boldsymbol{\Delta}): & =\int_{G} \int_{\mathbf{X}} f(g \boldsymbol{\Delta}) d \mathbf{x} d g=\int_{G}\left[\int_{\mathbf{X}} \sum_{i \in \mathbb{V}} h\left(g \boldsymbol{\Delta}, g \mathbf{x}_{i}\right) \delta\left(g \mathbf{x}-g \mathbf{x}_{i}\right) d \mathbf{x}\right] d g \\
& =\int_{G}\left[\sum_{i \in \mathbb{V}} h\left(\boldsymbol{\Delta}, \mathbf{x}_{i}\right)\right] d g=\sum_{i \in \mathbb{V}} h\left(\boldsymbol{\Delta}, \mathbf{x}_{i}\right) .
\end{aligned}
$$

Therefore we get invariants by simply adding local Euclidean-invariant (rotation and translation) DFLS $h\left(\boldsymbol{\Delta}, \mathbf{x}_{i}\right)$ (DFLSs Discrete Functions of Local $\underline{\text { Support) }}$ over all vertices. The interpretation of the result is very obvious: summing over locally Euclidean-invariants provides also global Euclidean invariants.

\subsection{Invariants for Polygons}

Let us apply the general principles for DHIs for polygons. As discrete functions of local support (DFLS) we choose monomials of the distances between neighboring vertices (which are obvious invariant to rotation) up to degree $k=4$ in the following example:

$$
\tilde{x}_{n_{1}, n_{2}, n_{3}, n_{4}}=\sum_{i \in \mathbb{V}} h\left(\boldsymbol{\Delta}, \mathbf{x}_{i}\right)=\sum_{i \in \mathbb{V}} d_{i, 1}^{n_{1}} d_{i, 2}^{n_{2}} d_{i, 3}^{n_{3}} d_{i, 4}^{n_{4}}
$$

and the $d_{i, k}$ denote the Euclidean distance of vertex $i$ and its $k$-th righthand neighbor:

$$
d_{i, k}=\left\|\mathbf{x}_{i}-\mathbf{x}_{<i+k>}\right\| .
$$

The brackets $<>$ denote a reduction modulo the number of vertices of a contour.

By varying the exponents we can build up a feature space. The nonlinear nature of this monomial-based kernel functions endow themselves with rich behaviors in the sense of discriminative ability. In general, the discrimination performance of this feature space will increase with the number of used features.

Principle of rigidity It can easily be shown that the features $\left\{d_{i, 1}, d_{i, 2}, d_{i, 3}\right\}$ (see Fig. 2) uniquely define a complete polygon (up to a mirror-polygon) because we can iteratively construct the polygon by rigidly piecing together rigid triangles 


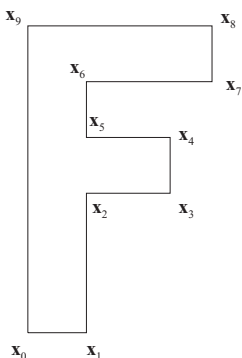

a)

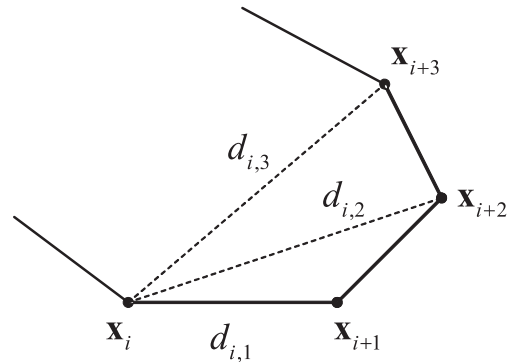

b)

Fig. 2. (a) Example of a polygon (b) basic features $\left\{d_{i, 1}, d_{i, 2}, d_{i, 3}\right\}$

(hereby we do not allow consecutive collinear edges in a polygon). Therefore we call these elements a basis. We expect to get a more and more complete feature space by integrating over more and more monomials from these basic features.

Looking at a triangle as the most simplest polygon one can show that the following three features derived from the three sides $\{a, b, c\}$ form a complete set of invariants:

$$
\tilde{x}_{0}=a+b+c, \quad \tilde{x}_{1}=a^{2}+b^{2}+c^{2}, \quad \tilde{x}_{2}=a^{3}+b^{3}+c^{3} .
$$

These features are equivalent to the elementary symmetrical polynomials in 3 variables which are a complete set of invariants with respect to all permutations.

It is not our intention to compare the results here with Fourier Descriptors (see e.g. [1]). First the approaches are rather different (integration over the transformation group versus normalization technique) and second the proposed method is much easier to extend from $2 \mathrm{D}$ to $3 \mathrm{D}$.

\subsection{D-Meshes}

Although we will not elaborate a rigorous concept for $3 \mathrm{D}$ discrete objects we want to outline that it is straightforward to extend the concept e.g to a 3DSurface mesh or a real 3D wireframe model. Again we derive DFLS of a certain neighborhood and sum over all vertices. It is appropriate to use here also basic features which meet the requirement that they constitute rigid local polyhedra which can rigidly pieced together to a solid surface or polyhedral object (see Fig. 31). Similar to the triangle as a basic building block for a planar polygon we can use a tetrahedron as a basic building block for a polyhedron. And as we can find three invariants for a triangle we similarly can find invariants for a tetrahedron derived from its edge lengths.

\subsection{Discrimination Performance and the Question of Completeness}

A crucial question is how many features we need to get a good discrimination performance and avoid ambiguities up to the point to use a complete set of 


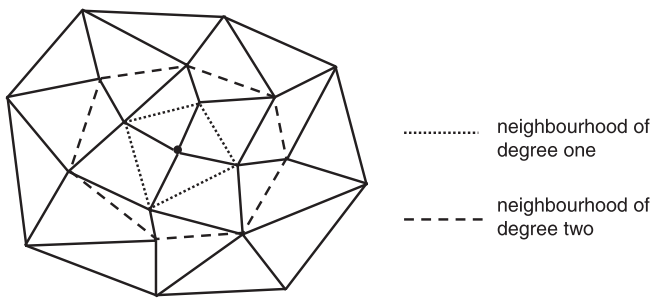

Fig. 3. Part of a surface mesh with neighborhood of degree one and two.

features. Asking for completeness is a very strong demand because it guarantees that no ambiguities between two different objects exist. For a practical pattern recognition problem we have to solve the much easier problem of separability to discriminate between a finite number of representatives of object classes (like the 26 classes in character recognition). Therefore we are typically content with a finite number of invariants which is typically much less than the number of features needed for completeness.

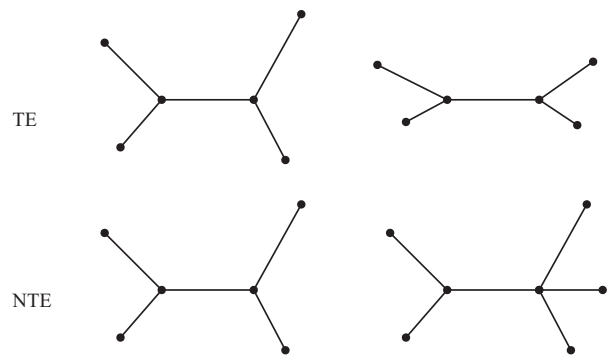

Fig. 4. Topologically equivalent (TE) and non topologically equivalent discrete structures (NTE).

As we use discrete features the methodology is sensitive to topological changes in the structure (see Fig. 4). So if we introduce another vertex on a polygon in the middle of an edge for example the invariants will clearly change. Discrete similarities are not necessarily visual similarities. Introducing an atom into a molecule also has a high impact on the function.

\section{Experiments: Object Classification in a Tangram Database}

We will demonstrate the construction of DHIs for the simplest case of closed polygons. As an example we have chosen a subset of 74 samples from the TangramMan-Database1 1 . Fig. [5 shows a selection of objects. As characteristic feature we

\footnotetext{
${ }^{1}$ see "Tangram Man" at http://www.reijnhoudt.nl/tangram
} 


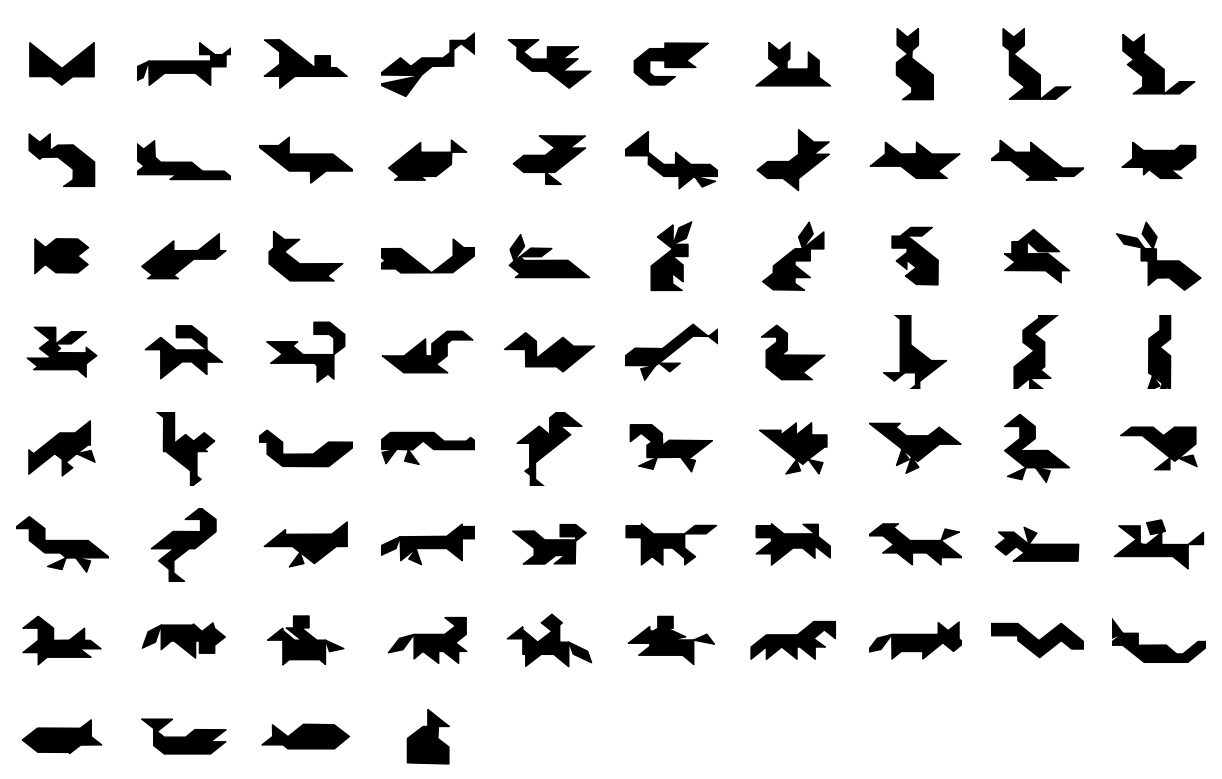

Fig. 5. The 74 tangrams used in the experiment.

extract the outer contour of the objects. This example is interesting because the objects can not easily be discriminated with trivial geometric features, as they all have the same area and their contours build clusters of rather few possible numbers.

As noise we added to each vertex a vector of a certain length with an uniformly distributed angle from $[0: 2 \pi]$. The amount of noise was measured against the standard deviation of all edges of the polygon $\sigma=\sqrt{\left(\frac{1}{|\mathbb{V}|}\left\|\mathbf{x}_{i}-\overline{\mathbf{x}}\right\|^{2}\right.}$. Fig. 6 shows an example with $10 \%$ noise added which leads already to remarkable distortions.

As the calculation of the monomials is a continuous function of the coordinates of the vertices we henceforth get a continuous propagation of the additive noise through the invariants. Therefore: little distortions will also cause little changes in the magnitude of the invariants.

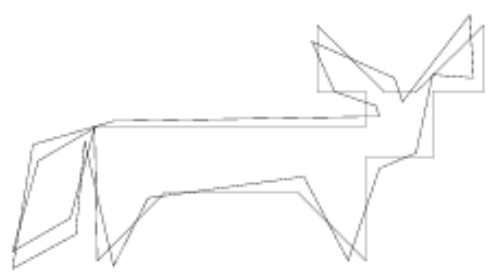

Fig. 6. Exact Contour and with $10 \%$ noise added. 
Table 1. Exponent table for monomials built from $\left\{d_{i, 1}^{n_{1}} d_{i, 2}^{n_{2}} d_{i, 3}^{n_{3}} d_{i, 4}^{n_{4}}\right\}$ for calculating 14 invariants

\begin{tabular}{|c|c|c|c|c|c|c|c|c|c|c|c|c|c|c|}
\hline $\mathrm{i}$ & $\tilde{x}_{0}$ & $\tilde{x}_{1}$ & $\tilde{x}_{2}$ & $\tilde{x}_{3}$ & $\tilde{x}_{4}$ & $\tilde{x}_{5}$ & $\tilde{x}_{6}$ & $\tilde{x}_{7}$ & $\tilde{x}_{8}$ & $\tilde{x}_{9}$ & $\tilde{x}_{10}$ & $\tilde{x}_{11}$ & $\tilde{x}_{12}$ & $\tilde{x}_{13}$ \\
\hline$n_{1}$ & 1 & 0 & 0 & 1 & 1 & 0 & 0 & 1 & 0 & 0 & 2 & 0 & 0 & 0 \\
$n_{2}$ & 0 & 1 & 0 & 1 & 0 & 1 & 0 & 0 & 1 & 0 & 0 & 2 & 0 & 0 \\
$n_{3}$ & 0 & 0 & 1 & 0 & 1 & 1 & 0 & 0 & 0 & 1 & 0 & 0 & 2 & 0 \\
$n_{4}$ & 0 & 0 & 0 & 0 & 0 & 0 & 1 & 1 & 1 & 1 & 0 & 0 & 0 & 2 \\
\hline
\end{tabular}

Table 2. Classification result for 5\%,10\% and $20 \%$ noise for 74 tangrams with an Euclidean (E) and a Mahalanobis (M) Classifier

\begin{tabular}{|c||ccccccccc|}
\hline noise (in percent) & 5 & 5 & 5 & 10 & 10 & 10 & 20 & 20 & 20 \\
\hline \# of invariants & 6 & 10 & 14 & 6 & 10 & 14 & 6 & 10 & 14 \\
\hline metric & $\mathrm{E}$ & $\mathrm{E}$ & $\mathrm{E}$ & $\mathrm{M}$ & $\mathrm{M}$ & $\mathrm{M}$ & $\mathrm{M}$ & $\mathrm{M}$ & $\mathrm{M}$ \\
\hline class. error (in percent) & 30 & 10 & 6 & 1.5 & 0 & 0 & 25 & 7 & 3 \\
\hline
\end{tabular}

The experiments were conducted with three sets of 6,10 and 14 invariants respectively according to eq. (6) (see Table 1) (the subset of 6 and 10 are just the first 6 and 10 of the 14 invariants. The classification performance was measured against additive noise of $5 \%, 10 \%$ and $20 \%$.

Table 2 shows the result of our experiments averaged over 50 samples. Choosing for the sake of simplicity an Euclidean classifier measuring the distance to the expectation of the class centers leads to rather bad classification results. Adding only $5 \%$ noise results in a misclassification of $30 \%$ of the tangrams using 6 invariants. This number can be reduced to $10 \%$ false classifications adding further 4 invariants and to $6 \%$ using 14 invariants. This result is not surprising. Looking at the invariants we can observe a large variance of their magnitudes due to the differences in the degree of the monomials. We can, however, drastically improve the result by using a Mahalanobis-Classifier. This is due to the fact that the noise covariance matrices of all objects are very similar. Therefore we made experiments with a Mahalanobis-Classifier based on an averaged covariance matrix over all object classes. Now even an increase of noise by a factor two to $10 \%$ leads only to $1.5 \%$ errors with 6 invariants and $0 \%$ errors for 10 and 14 invariants which demonstrates the very good performance of the invariants. Even with $20 \%$ noise and 14 invariants we get a rather low error rate of only $3 \%$.

If we constrain our calculation to a finite number of invariants we end up with a simple linear complexity in the number of vertices. This holds if the local neighborhood of vertices is resolved already by the given data structure; otherwise the cost for resolving local neighborhoods must be added. In contrast to graph matching algorithms we apply here algebraic techniques to solve the problem. This has the advantage that we can apply hierarchical searches for 
retrieval tasks, namely, to start only with one feature and hopefully eliminate already a large number of objects and then continue with an increasing number of features etc.

\section{Conclusions}

In this paper we have introduced a novel set of invariants for discrete structures in $2 \mathrm{D}$ and $3 \mathrm{D}$. The construction is a rigorous extension of Haar integrals over transformation groups to Dirac Delta Functions. The resulting invariants can easily be calculated with linear complexity in the number of vertices. The proposed approach has the potential to be extended to other discrete structures and even to the more general case of weighted graphs.

Acknowledgement. This paper has been worked out while the first author was on a sabbatical leave at the National ICT Australia (NICTA), Department of Systems Engineering, The Australian National University (ANU), Canberra ACT, Australia.

We are very thankful to Mr. Frans Reijnhoudt who allowed us to use his Tangram-Database. We also thank Bernard Haasdonk and the anonymous reviewers for their helpful comments and suggestions.

\section{References}

1. K. Arbter, W. Snyder, H. Burkhardt, and G. Hirzinger. Application of AffineInvariant Fourier Descriptors to 3-D Objects. IEEE Trans. on Pattern Analysis and Machine Intelligence, PAMI-12(7):640-647, July 1990.

2. H. Burkhardt and S. Siggelkow. Invariant features in pattern recognition - fundamentals and applications. In C. Kotropoulos and I. Pitas, editors, Nonlinear Model-Based Image/Video Processing and Analysis, pages 269-307. John Wiley \& Sons, 2001.

3. O. Ronneberger, H. Burkhardt, and E. Schultz. General-purpose Object Recognition in 3D Volume Data Sets using Gray-Scale Invariants - Classification of Airborne Pollen-Grains Recorded with a Confocal Laser Scanning Microscope. In Proceedings of the International Conference on Pattern Recognition, Quebec, Canada, September 2002.

4. H. Schulz-Mirbach. On the Existence of Complete Invariant Feature Spaces in Pattern Recognition. In Proc. of the 11th International Conference on Pattern Recognition, Conference B: Pattern Recognition Methodology and Systems, volume II, pages 178-182, Den Haag, 1992.

5. H. Schulz-Mirbach. Anwendung von Invarianzprinzipien zur Merkmalgewinnung in der Mustererkennung. PhD thesis, Technische Universität Hamburg-Harburg, February 1995. Reihe 10, Nr. 372, VDI-Verlag.

6. H. Schulz-Mirbach. Invariant features for gray scale images. In G. Sagerer, S. Posch, and F. Kummert, editors, 17. DAGM - Symposium "Mustererkennung", pages 1-14, Bielefeld, 1995. Reihe Informatik aktuell, Springer. DAGM-Preis. 